\title{
Société suisse de Chirurgie Plastique, Reconstructive et Esthétique (SSCPRE)
}

\author{
Pierre Schertenleib ${ }^{a}$, \\ Catherine Perrin ${ }^{b}$ \\ a Dr méd., président \\ b directrice
}

1 Verdan C. Histoire de la Société suisse de Chirurgie plastique, reconstructive et esthétique; 1985.

Correspondance:

Catherine Perrin, PhD Office SGRPAC-SSCPRE 15, avenue des Planches CH-1820 Montreux

info@plastic-surgery.ch www.plastic-surgery.ch

\section{Création et but}

La Société Suisse de Chirurgie Plastique, Reconstructive et Esthétique (SSCPRE) est l'association professionnelle des médecins spécialistes de cette discipline. La société a été créée en 1965 à Locarno par ses membres fondateurs Federico Andina (président), Rodolphe Meyer, Hans-Ueli Buff, Claude Koechlin et Peter Ricklin [1]. La Société a pour but la promotion de la spécialité dans le domaine de la recherche et de l'enseignement et entretient des relations avec les sociétés et organisations apparentées suisses ou étrangères.

La Société défend ses intérêts professionnels et économiques et veille au respect des principes éthiques de l'activité médicale de ses membres selon le code de déontologie de la FMH. La Société reconnaît les statuts de la Fédération des Médecins Suisses FMH. Elle est responsable de la formation postgraduée et continue et définit le contenu des programmes respectifs en contrôlant leur mise en œuvre avec le souci de préserver l'ensemble du spectre d'activité relevant de la spécialité. Une fois par an, elle organise un congrès scientifique, en règle générale le premier week-end du mois d'octobre dont le programme couvre tous les domaines de la discipline (chirurgie de la main, plastie, reconstruction, esthétique). Les jeunes assistants en formation ont la possibilité de présenter les résultats de leurs recherches. La Société compte 200 membres, dont 133 ordinaires. Elle croît chaque année en moyenne de cinq membres.

\section{Assurance de qualité}

Depuis quelques années déjà, la Société se fait un devoir d'être active dans le domaine de l'assurance de qualité. Elle a élaboré des protocoles d'information (informed consent) qui permettent au médecin d'informer le patient d'une manière approfondie sur l'intervention ainsi que sur ses risques. A partir de juillet 2009, les implants mammaires sont répertoriés dans un registre national. Dès cette date également les membres seront invités à enregistrer leurs interventions. Cette statistique permettra dans un premier temps un bench-

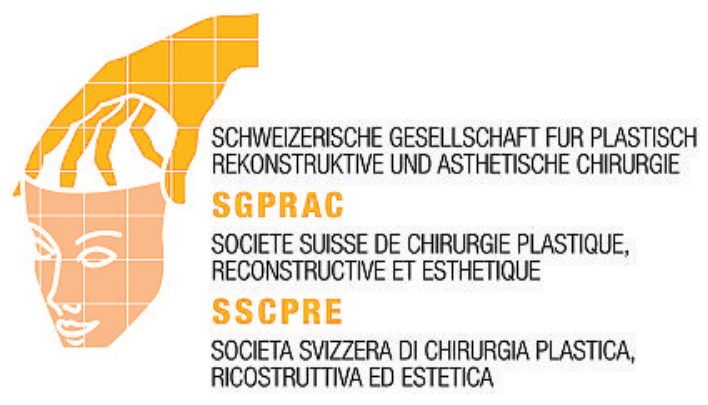

marking numérique. Il est prévu que dans un ou deux ans, non seulement les interventions soient enregistrées, mais également les révisions, les complications, les infections, etc. Cela permettra alors un benchmarking et des conclusions sur la qualité des interventions, du diagnostic et du suivi. Ces instruments de qualité ne resteront pas lettre morte; ils représentent un premier pas pour plus de sécurité pour le patient et permettent au corps médical l'analyse de son activité médicale.

\section{Information}

Depuis l'essor de la chirurgie esthétique, la Société s'est fait un devoir d'informer la population des risques de ces interventions. C'est ainsi qu'un nouveau site Internet a permis d'améliorer très nettement l'information. La discipline y est décrite, la plupart des interventions expliquées; il met en garde contre les risques d'offres alléchantes et aux prix cassés, d'opérations faites à l'étranger. Il contient de plus la liste de tous les médecins plasticiens actifs et membres de la Société. Celle-ci, en complément au site Internet, a publié une brochure pour les patients «Tout ce que vous devez savoir sur la Chirurgie Plastique, Reconstructive et Esthétique», un petit guide, téléchargeable sur le site www.plastic-surgery.ch. Il peut aussi être commandé par téléphone au 0219632139 ou par courriel info@ plastic-surgery.ch. Que ce soit le site Internet ou encore la brochure, les deux instruments sont hautement appréciés et utilisé par un grand nombre de personnes. 